\title{
The prognostic role of long noncoding RNA CRNDE in cancer patients: a systematic review and meta-analysis
}

\author{
W. WANG*, F. YUAN*, J. XU* \\ Department of Breast Surgery, Hubei Cancer Hospital, Tongji Medical College, Huazhong University of Science and Technology, Wuhan, Hubei \\ 430079, P.R. China
}

${ }^{*}$ Correspondence: doctorxujuan@aliyun.com

"Contributed equally to this work.

Received March 20, 2018 / Accepted June 18, 2018

\begin{abstract}
The novel colorectal neoplasia differentially expressed lncRNA (CRNDE) has been discovered in many solid tumors. We conducted this systematic review and meta-analysis to illuminate the function of CRNDE in cancer. Published articles were identified by electronic search of PubMed, EMBASE, and MEDLINE using the keywords "cancer or carcinoma or neoplasm or tumor" and "long noncoding RNA CRNDE or noncoding RNA CRNED or CRNDE". Hazard ratios (HRs), standard errors (SEs) and their corresponding 95\% confidence intervals (CIs) were estimated from study survival curves. Odds ratios (ORs) with 95\% CIs were estimated in order to evaluate the correlation between CRNDE expression level and cancer patient clinical outcome. Herein, 13 of the 49 identified studies completely fulfilled inclusive criteria for this systemic review. The results are as follows: the pooled HR of high expression versus low expression of CRNDE was 1.64 with a $95 \%$ CI of $[1.44,1.87]$; the pooled OR of high CRNDE expression in early TNM stages versus late TNM stages was 0.35 with a $95 \%$ CI of $[0.26,0.47]$; the pooled OR of high CRNDE expression in positive lymph node metastasis versus negative lymph node metastasis was 3.62 with a $95 \%$ CI of $[1.99,6.58]$; the pooled OR of high CRNDE expression in tumor size larger than $5 \mathrm{~cm}$ versus tumor size smaller than $5 \mathrm{~cm}$ was 2.99 with a $95 \% \mathrm{CI}$ of $[1.13,7.93]$. For colorectal cancer studies, the pooled OR of high CRNDE expression in colon tumor versus rectal tumor was 1.18 with a $95 \%$ CI of $[0.64,2.18]$, the pooled OR of high CRNDE expression among T1 and T2 versus T3 and T4 was 0.91 with a 95\% CI of $[0.57,1.46]$, and the pooled OR of high CRNDE expression in colorectal cancer patients without distant metastasis versus patients with distant metastasis was 0.48 with a $95 \%$ CI of $[0.21,1.10]$. No obvious publication bias was observed in any study in this meta-analysis.

CRNDE was confirmed an oncogene, and its up-regulated expression significantly correlated with poor prognosis and advanced tumor progression.
\end{abstract}

Key words: CRNDE, prognosis, systematic review, meta-analysis

Despite advances in modern medicine, cancer remains a primary global cause of mortality. Oncologists should first consider patient survival, and improvements in early diagnosis and treatment can significantly improve patient survival. During the past few decades, many proteins have been proposed as effective cancer biomarkers at the laboratory research level [1]. However, few of these have been reliably tested in clinical settings. When confronted with the entire human genome, oncological researchers should therefore consider diverting their attention to regions outside protein coding genes to find reliable cancer diagnostic and prognostic markers.

There are thousands of non-coding regions in the human genome, but due to a lack of evidence that they are transcribed or that they encode a protein, for over a decade these non-coding regions were considered "junk DNA". Recent advances in genetic technology established that up to $75 \%$ of the human genome could be transcribed into RNAs, with less than $2 \%$ encoding proteins [2] in the large human transcriptome,

Long noncoding RNAs (lncRNAs) have over 200 nucleotides but do not code proteins $[3,4]$, and their discovery provides a significant new perspective in genetic regulation.

The novel lncRNA, colorectal neoplasia differentially expressed (CRNDE), was originally identified as an overregulated gene in colorectal cancers, with low or no expression in normal colon epithelia cells [5]. This encouraged investigation into serum-based CRNDE as a biomarker for 
colorectal cancer diagnosis and prognosis [6]. Moreover, CRNDE was discovered in many other solid tumors, including breast cancer [7], gastric cancer [8], glioma [9], liver cancer [10], lung cancer [11], multiple myeloma [12] and pancreatic cancer [13]. CRNDE is a presumed oncogene, promoting cancer cell growth and metastasis [14-18] and is also believed to have a role in oxaliplatin-based chemotherapy resistance [16]. Determining how CRNDE performs as an oncogene will help improve therapeutic treatment and patient survival. These studies, however, were restricted by sample volume limits and research bias and this restricted full investigation into the implications of CRNDE expression. We therefore conducted this systematic review and meta-analysis to illuminate CRNDE functions in cancer.

\section{Material and methods}

Literature search. Articles were identified by electronic search of PubMed, EMBASE, and MEDLINE using the keywords "cancer, carcinoma, neoplasm, tumor" and "long non-coding RNA CRNDE and non-coding RNA CRNED and CRNDE". These key words were applied in different combinations. The bibliographies from identified studies were used to identify further trials. The articles are limited to published trials, with at least the abstract given in English. No contact was made with the authors to obtain unpublished data.

Selection of trials. A total of 49 articles were obtained from January 2007 to January 2018. Inclusion criteria were determined before finalizing candidate articles. The potential literature to be included in this review had to fulfil the

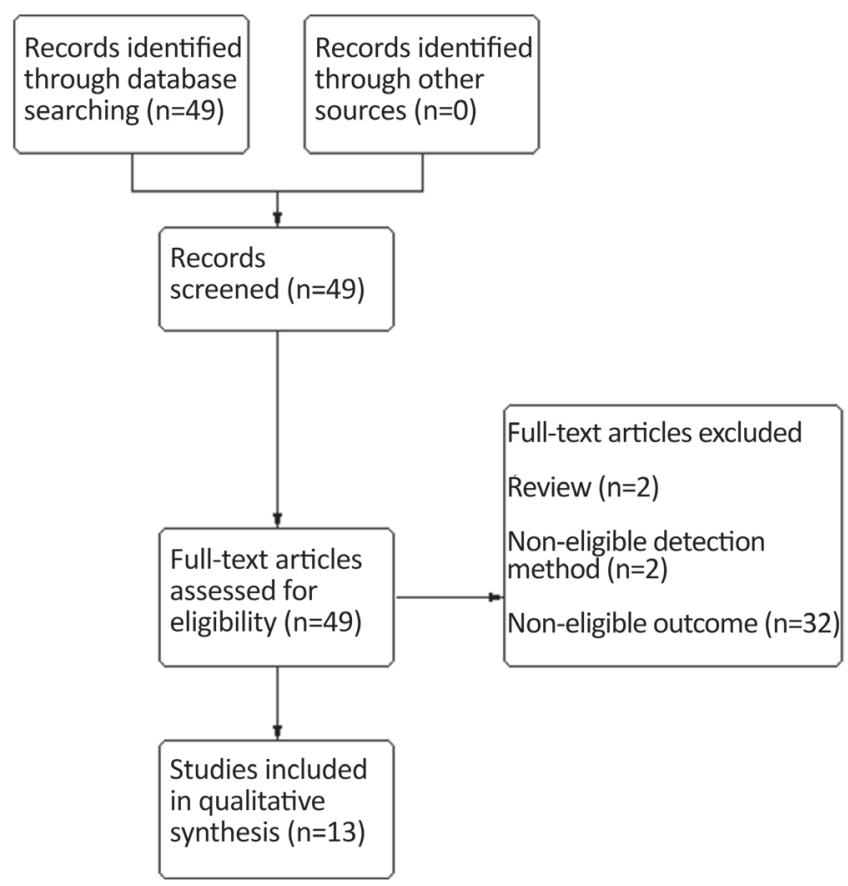

Figure 1. Study flow diagram. following criteria: (1) the research contained at least one of the following clinical characteristics: overall survival (OS), tumor size, lymph node metastasis, TNM stage or pathological stage; (2) the expression of long noncoding RNA CRNDE was evaluated in tumor tissues only (not in serum or other tissues), and the method was qualitative real-time PCR (qRT-PCR) or in situ hybridization (ISH); (3) all samples were extracted from humans and (4) all the patients were divided into groups of high and low CRNDE expression. The quality of candidate studies was assessed via the NewcastleOttawa Scale (NOS) standard which included selection (4 points), comparability ( 2 points) and outcome (3 points), with a score range of $0 \sim 9$. A study with NOS score of 6 or more was included.

Data extraction and quality assessment. Two investigators independently extracted the data from each original publication, including the first author's name, the year of publication, the country of origin, the cancer type, the total number of patients and those in the high and low CRNDE expression groups, the detection method, the outcome measures and the cut-off value for CRNDE levels. Missing information was estimated according to the Cochrane Handbook and was requested from the authors of the original studies if necessary. Discrepancies between the 2 investigators were resolved by discussion and consensus.

Although survival curves were available, only 11 of the 13 selected studies provided Kaplan-Meier curves and none gave the HRs and corresponding 95\% CIs. Using widely proven and accepted scientific methodology, data was extracted from the survival curves with Engauge 4.1 (http:// digitizer.sourceforge.net/) [19-21]. This data formed input for the HR calculation spreadsheet created by Tierney et al. [22]. The HRs, SEs and their corresponding 95\% CIs then were estimated from the curves.

Statistical analysis. The ORs with $95 \%$ CIs were estimated in order to evaluate correlation between the expression level of long non-coding RNA CRNDE and cancer patient clinical outcome. TNM stages were separated into early stage (I-II) and advanced stage (III-IV), as in the American Joint Committee on Cancer (AJCC) staging system, and lymph node metastasis (LNM) was divided into "positive" and "negative" groups. All extracted data was pooled in Review Manager 5.3 (The Nordic Cochrane, Denmark), and statistical heterogeneity was defined as $\mathrm{P}<0.10$ or $\mathrm{I}^{2}>50 \%$ according to previously reported experience [21]. If heterogeneity occurred, a randomized effect model was used to pool the results; otherwise, a fixed effect model was applied.

\section{Results}

Characteristics of the included trials. Figure 1 shows that 13 of the 49 studies originally identified completely fulfilled inclusive criteria for this systematic review. Studies 7 , $8,10-13,23-29$ covered 8 kinds of cancers: 1 in breast cancer [7], 4 in colorectal cancer [23-26], 1 in gastric cancer [8], 3 in 
Table 1. Characteristics of studies included in this meta-analysis.

\begin{tabular}{|c|c|c|c|c|c|c|c|c|c|c|}
\hline \multirow{2}{*}{ First author } & \multirow{2}{*}{ Year } & \multirow{2}{*}{ Country } & \multirow{2}{*}{ Cancer type } & \multirow{2}{*}{$\begin{array}{l}\text { Total } \\
\text { cases }\end{array}$} & \multicolumn{2}{|c|}{ CRNDE expression } & \multirow{2}{*}{$\begin{array}{l}\text { Detection } \\
\text { method }\end{array}$} & \multirow{2}{*}{$\begin{array}{l}\text { Outcome } \\
\text { method }\end{array}$} & \multirow{2}{*}{$\begin{array}{c}\text { Cut-off } \\
\text { (high/low) }\end{array}$} & \multirow{2}{*}{$\begin{array}{c}\text { Quality } \\
\text { score }\end{array}$} \\
\hline & & & & & High & Low & & & & \\
\hline HUAN & 2017 & China & Breast Cancer & 103 & 44 & 59 & qRT-PCR & Overall Survival & $\mathrm{N} / \mathrm{A}$ & 6 \\
\hline DING & 2017 & China & Colorectal Cancer & 80 & 40 & 40 & qRT-PCR & N/A & Median & 6 \\
\hline HAN & 2017 & China & Colorectal Cancer & 64 & 32 & 32 & qRT-PCR & Overall Survival & Median & 7 \\
\hline JIANG & 2017 & China & Colorectal Cancer & 251 & 120 & 131 & ISH & Overall Survival & score $\geq 3$ & 6 \\
\hline YU & 2017 & China & Colorectal Cancer & 21 & 10 & 11 & qRT-PCR & N/A & Median & 7 \\
\hline DU & 2017 & China & Gastric Cancer & 118 & 61 & 57 & qRT-PCR & Overall Survival & Median & 8 \\
\hline JING & 2016 & China & Glioma & 164 & 83 & 81 & qRT-PCR & Overall Survival & Median & 7 \\
\hline GRAVENDEEL & 2009 & Netherlands & Glioma & 101 & 51 & 50 & qRT-PCR & Overall Survival & Median & 6 \\
\hline MURAT & 2008 & Switzerland & Glioma & 68 & 34 & 34 & qRT-PCR & Overall Survival & Median & 6 \\
\hline DAI & 2017 & China & Liver Cancer & 370 & 184 & 186 & qRT-PCR & Overall Survival & $\mathrm{N} / \mathrm{A}$ & 7 \\
\hline ZHANG & 2017 & China & Lung Cancer & 66 & 36 & 30 & qRT-PCR & Overall Survival & $\mathrm{N} / \mathrm{A}$ & 6 \\
\hline MENG & 2017 & China & Multiple Myeloma & 77 & 47 & 30 & qRT-PCR & Overall Survival & $\mathrm{N} / \mathrm{A}$ & 7 \\
\hline WANG & 2017 & China & Pancreatic Cancer & 58 & 38 & 20 & qRT-PCR & Overall Survival & $\mathrm{N} / \mathrm{A}$ & 6 \\
\hline
\end{tabular}

glioma [27-29], 1 in liver cancer [10], 1 in lung cancer [11], 1 in multiple myeloma [12] and 1 in pancreatic cancer [13].]. The remaining 36 trials were excluded because of study type, ineligible detection method or ineligible outcomes.

Table 1 reveals that the 13 eligible trails were from China and Europe and that they involved 1,541 total patients; 780 with high CRNDE expression and 761 with low expression. The expression level of CRNDE in the patients was evaluated by qRT-PCR or ISH analysis. Eleven studies provided overall survival curves and seven applied the median CRNDE expression level as the cut-off for grouping; but cut-off values for the remaining 6 studies were unavailable.

Association between CRNDE expression and overall survival. A total of 11 studies reported the influence of CRDNE expression on overall survival. Figure 2 highlights that the fixed effect model evaluated the pooled HR and 95\% CI because of the lack of significant heterogeneity $(\mathrm{p}=0.15$, $\mathrm{I}^{2}=31 \%$ ). The pooled HR of high CRNDE expression over low expression was 1.64 with a $95 \%$ CI of $[1.44,1.87]$. This indicated that highly expressed CRNDE was associated with impaired overall survival rate in these cancer patients.

There were several kinds of cancer patients in this review, so subgroup meta-analysis estimated the influence of high CRNDE expression on overall survival rate in different cancers. Up-regulated CRNDE was related to low survival rate for the following cancers: breast (HR 1.90, 95\% CI [1.05, $3.41]$ ), gastric (HR 2.05, 95\% CI [1.16, 3.63]), glioma (HR $1.70,95 \%$ CI $[1.33,2.18]$ ), liver (HR 1.62, 95\% CI [1.28, 2.04]), lung (HR 2.80, 95\% CI $[1.59,4.95])$ and multiple myeloma (HR 1.90, 95\% CI [1.05, 3.41]). However, there was no significant difference between CRNDE expression and overall survival in colorectal cancer (HR 1.12, 95\% CI [0.81, 1.56]) or pancreatic cancer (HR 1.75, 95\% CI [0.94, 3.28]).

Association between CRNDE expression and TNM stage. Nine of the thirteen chosen studies reported the influence of CRDNE expression on TNM stage. Figure 3 shows that the fixed effect model evaluated the pooled OR and 95\% CI due to significant heterogeneity $\left(\mathrm{p}=0.11, \mathrm{I}^{2}=39 \%\right)$. The pooled OR of high CRNDE expression in early TNM stages versus late TNM stages was 0.35 with a $95 \%$ CI of $[0.26,0.47]$. This indicates that highly expressed CRNDE is associated with advanced TNM stages in these cancers.

Subgroup meta-analysis then estimated the influence of high CRNDE expression on TNM stages in different cancers, because different cancers featured in this review. Up-regulated CRNDE was related to advanced TNM stage for the following cancers: breast (OR $0.33,95 \%$ CI [0.14, $0.81])$, colorectal (OR $0.14,95 \%$ CI $[0.06,0.32])$, gastric (OR $0.38,95 \%$ CI $[0.18,0.80]$ ), glioma (OR $0.23,95 \%$ CI [0.11, $0.50]$ ), lung (OR $0.30,95 \%$ CI $[0.10,0.95])$ and pancreatic cancer (OR 0.22, 95\% CI $[0.07,0.72]$ ). There was, however, no significant difference between CRNDE expression and TNM stage for liver cancer (OR $0.66,95 \%$ CI $[0.41,1.07])$.

Association between CRNDE expression and lymph node metastasis. A total of 6 studies reported the influence of CRDNE expression on lymph node metastasis, and Figure 4 depicts the randomized effect model applied to evaluate the pooled OR and $95 \%$ CI because of significant heterogeneity $\left(p=0.05, I^{2}=56 \%\right)$. The pooled OR of high CRNDE expression in positive and negative lymph node metastasis was 3.62 with a $95 \%$ CI of $[1.99,6.58]$. This indicates that highly expressed CRNDE was associated with lymph node metastasis in these cancer patients.

Subgroup meta-analysis then estimated the influence of high CRNDE expression on lymph node metastasis in the different cancers. Up-regulated CRNDE was significantly related to lymph node metastasis for the following cancers: colorectal (OR 2.37, 95\% CI [1.21, 4.64]), gastric (OR 2.92, $95 \%$ CI $[1.37,6.20])$, lung (OR 11.50, 95\% CI [3.62, 36.49]) and pancreatic cancer (OR 5.06, 95\% CI [1.56, 16.38]).

Association between CRNDE expression and colorectal cancer. Figure 5 reveals that three studies reported the influ- 


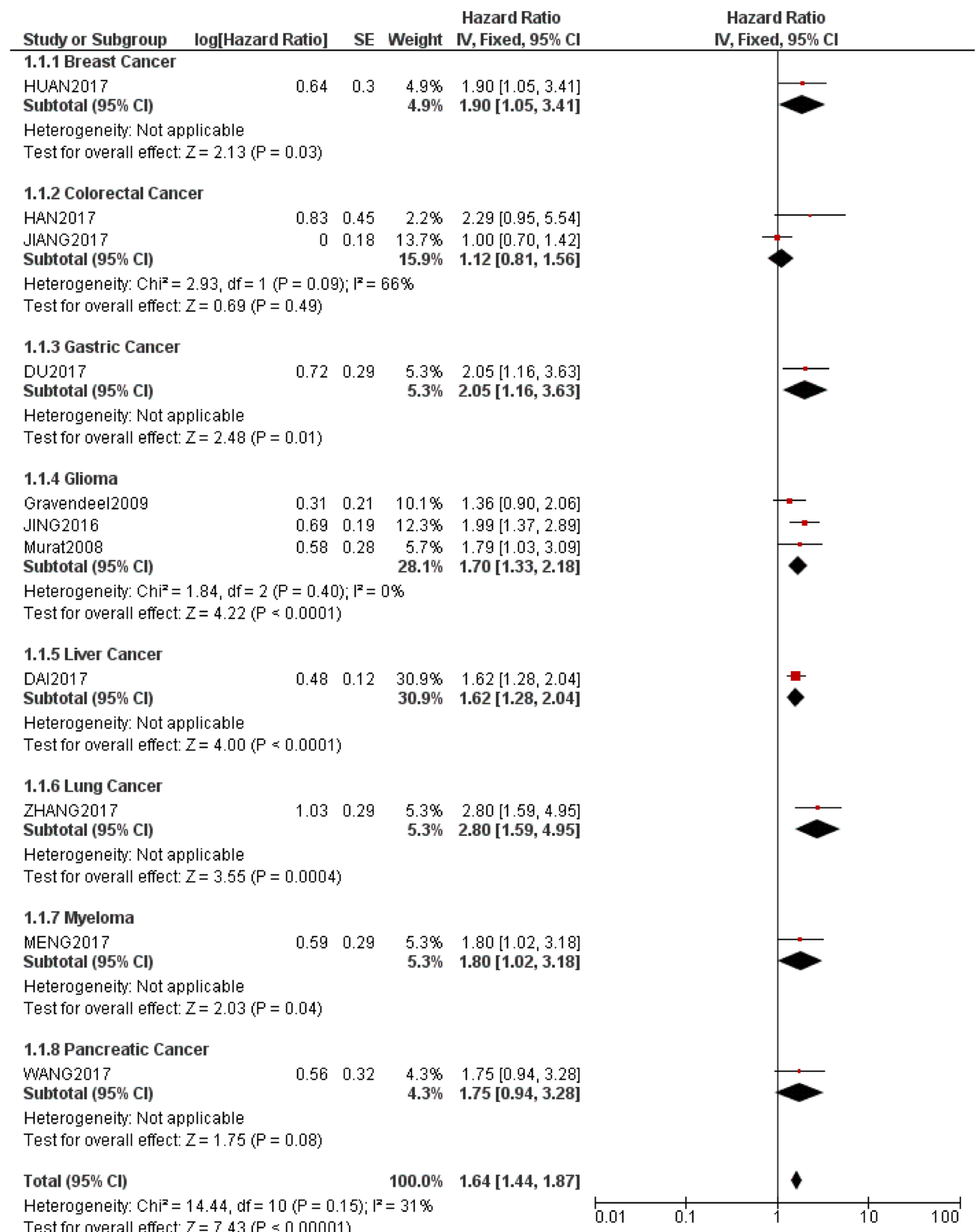

Test for subaroup differences: $\mathrm{Ch}^{2}=9.67, \mathrm{df}=7(\mathrm{P}=0.21), \mathrm{I}^{2}=27.6 \%$

Figure 2. CRNDE and OS. Forest plot of HRs for the association between CRNDE expression and OS. 


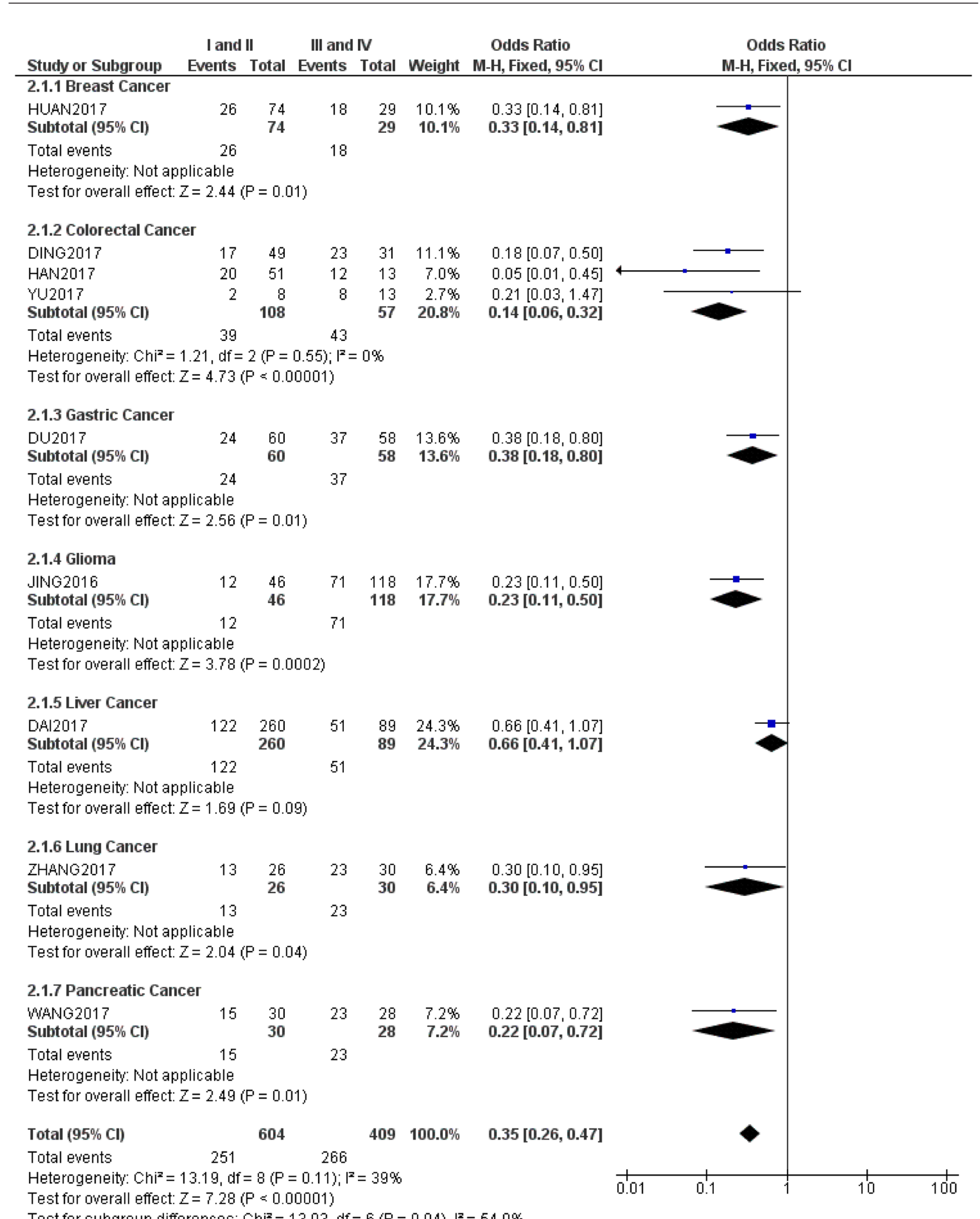

Figure 3. CRNDE and TNM. Forest plot of ORs for the association between CRNDE expression and TNM stage. 


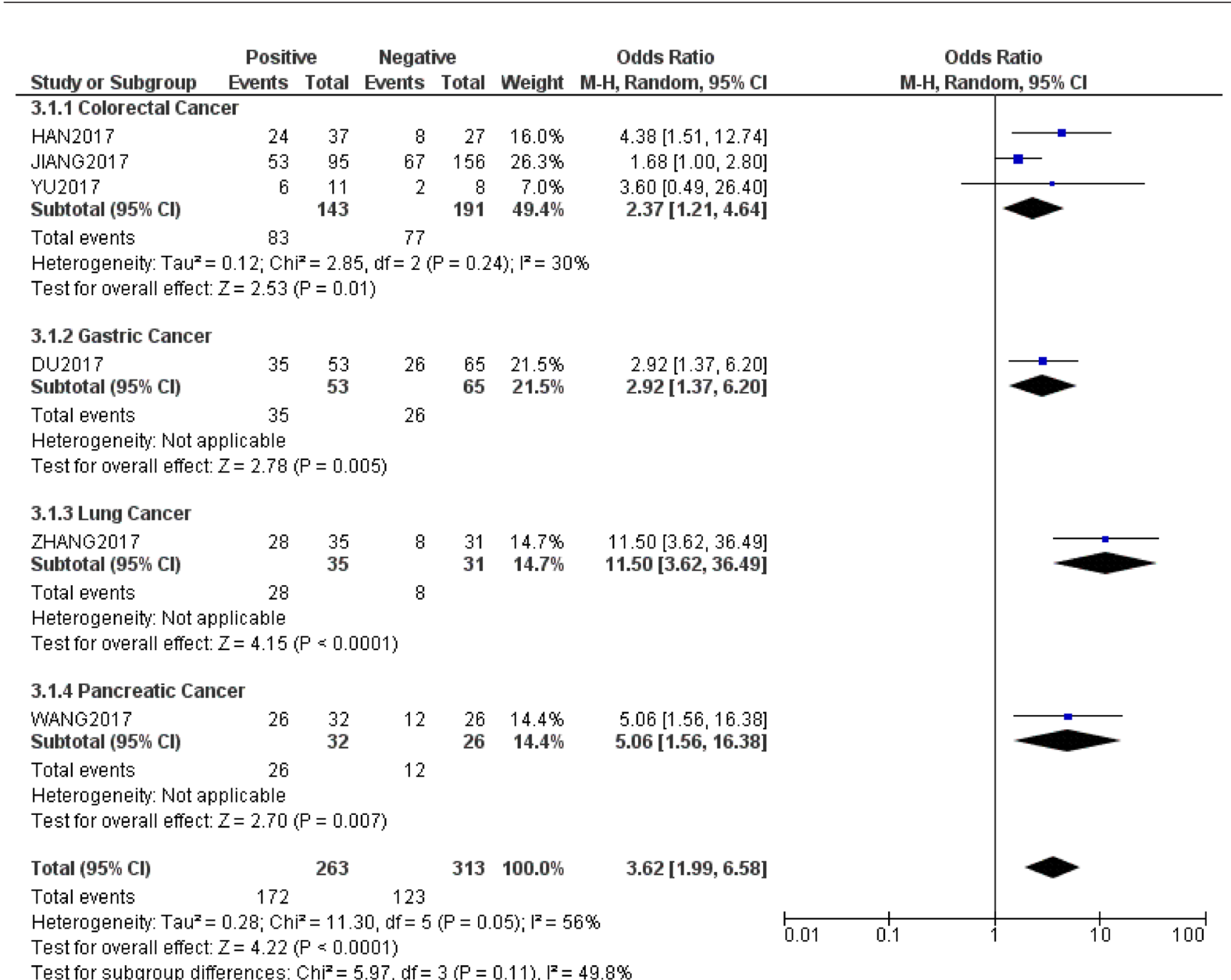

Figure 4. CRNDE and lymph node metastasis. Forest plot of ORs for the association between CRNDE expression and lymph node metastasis.

ence of CRDNE expression on tumor size in colorectal cancer. Here, the randomized effect model evaluated the pooled OR and $95 \%$ CI because of the resultant significant heterogeneity $\left(\mathrm{p}=0.02, \mathrm{I}^{2}=76 \%\right)$. The pooled OR of high CRNDE expression in tumor size larger and smaller than $5 \mathrm{~cm}$ was 2.99 with a $95 \%$ CI of $[1.13,7.93]$, and this indicated that highly expressed CRNDE was associated with larger tumor size in colorectal cancer patients.

Figure 6 depicts that three studies reported the influence of CRDNE expression on tumor site in colorectal cancer. The fixed effect model evaluated the pooled OR and $95 \%$ CI because there was no significant heterogeneity $(p=0.34$, $\mathrm{I}^{2}=8 \%$ ). The pooled OR of high CRNDE expression in colon tumor versus rectal tumor was 1.18 with a $95 \%$ CI of [0.64, 2.18], thus indicating no correlation between CRNDE expression and tumor site in colorectal cancer patients.
Further, Figure 7 shows that three studies reported the influence of CRDNE expression on colorectal cancer $\mathrm{T}$ stage. The fixed effect model evaluated pooled OR and 95\% CI. because of the lack of resultant significant heterogeneity $\left(\mathrm{p}=0.18, \mathrm{I}^{2}=41 \%\right)$. The pooled OR of high CRNDE expression among T1 and T2 versus T3 and T4 was 0.91 with a 95\% CI of $[0.57,1.46]$, thus indicating no correlation between CRNDE expression and $\mathrm{T}$ stage for colorectal cancer patients.

As shown in Figure 8, three studies reported the influence of CRDNE expression on distant colorectal metastases, and here the fixed effect model evaluated pooled OR and 95\% CI due to no significant heterogeneity $\left(p=0.25, I^{2}=28 \%\right)$. The pooled OR of high CRNDE expression in patients with and without distant metastasis was 0.48 with a $95 \%$ CI of $[0.21$, 1.10], thus indicating no correlation between CRNDE expression and distant metastasis in colorectal cancer patients. 


\begin{tabular}{|c|c|c|c|c|c|c|c|c|}
\hline \multirow[b]{2}{*}{ Study or Subgroup } & \multicolumn{2}{|c|}{$\geq 5 \mathrm{~cm}$} & \multicolumn{2}{|c|}{$<5 \mathrm{~cm}$} & \multirow[b]{2}{*}{ Weight } & $\begin{array}{c}\text { Odds Ratio } \\
\text { Pandom }\end{array}$ & \multirow{2}{*}{$\begin{array}{c}\text { Odds Ratio } \\
\text { M-H, Random, } 95 \% \mathrm{Cl}\end{array}$} & \\
\hline & Events & Total & Events & Total & & M-H, Random, $95 \% \mathrm{Cl}$ & & \\
\hline DING2017 & 25 & 36 & 15 & 44 & $31.4 \%$ & $4.39[1.71,11.30]$ & & \\
\hline HAN2017 & 24 & 35 & 8 & 29 & $28.8 \%$ & $5.73[1.94,16.91]$ & & \\
\hline JANG2017 & 77 & 151 & 43 & 100 & $39.8 \%$ & $1.38[0.83,2.29]$ & & \\
\hline Total $(95 \% \mathrm{Cl})$ & & 222 & & 173 & $100.0 \%$ & $2.99[1.13,7.93]$ & & \\
\hline Total events & 126 & & 66 & & & & & \\
\hline $\begin{array}{l}\text { Heterogeneity: Tau } \\
\text { Test for overall effect }\end{array}$ & $\begin{array}{l}0.55 ; \mathrm{Chi} \\
Z=2.20(\end{array}$ & $\begin{array}{l}=8.27 \\
P=0.0\end{array}$ & , df $=26$ & $P=0.0$ & $2) ; 1^{2}=76^{\circ}$ & & $\begin{array}{ll}1 \\
0.01 & 0.1\end{array}$ & 100 \\
\hline
\end{tabular}

Figure 5. CRNDE and tumor size. Forest plot of ORs for the association between CRNDE expression and colorectal tumor size.

\begin{tabular}{|c|c|c|c|c|c|c|c|}
\hline \multirow[b]{2}{*}{ Study or Subgroup } & \multicolumn{2}{|c|}{ Colon } & \multicolumn{2}{|c|}{ Rectum } & \multirow[b]{2}{*}{ Weight } & \multirow{2}{*}{$\begin{array}{c}\text { Odds Ratio } \\
\text { M-H, Fixed, } 95 \% \text { Cl }\end{array}$} & \multirow{2}{*}{$\begin{array}{c}\text { Odds Ratio } \\
\text { M-H, Fixed, } 95 \% \text { Cl }\end{array}$} \\
\hline & Events & Total & Events & Total & & & \\
\hline DING2017 & 18 & 33 & 22 & 47 & $44.3 \%$ & $1.36[0.56,3.33]$ & \\
\hline HAN2017 & 17 & 31 & 15 & 33 & $35.2 \%$ & $1.46[0.54,3.90]$ & \\
\hline YU2017 & 2 & 7 & 8 & 14 & $20.5 \%$ & $0.30[0.04,2.11]$ & \\
\hline Total $(95 \% \mathrm{CI})$ & & 71 & & 94 & $100.0 \%$ & $1.18[0.64,2.18]$ & \\
\hline Total e'vents & 37 & & 45 & & & & \\
\hline $\begin{array}{l}\text { Heterogeneity: Chi }= \\
\text { Test for overall effect }\end{array}$ & $\begin{array}{l}2.17, \mathrm{df}= \\
z=0.52 \mathrm{l}\end{array}$ & $\begin{array}{l}2(P= \\
P=0.6\end{array}$ & $\begin{array}{l}0.34) ; 1^{2}= \\
i 0)\end{array}$ & $8 \%$ & & & $0.01 \quad 0.1$ \\
\hline
\end{tabular}

Figure 6. CRNDE and tumor site. Forest plot of ORs for the association between CRNDE expression and colorectal tumor site.

T1 and T2 T3 and T4 Odds Ratio Odds Ratio

Study or Subgroup Events Total Events Total Weight M-H, Fixed, 95\% Cl

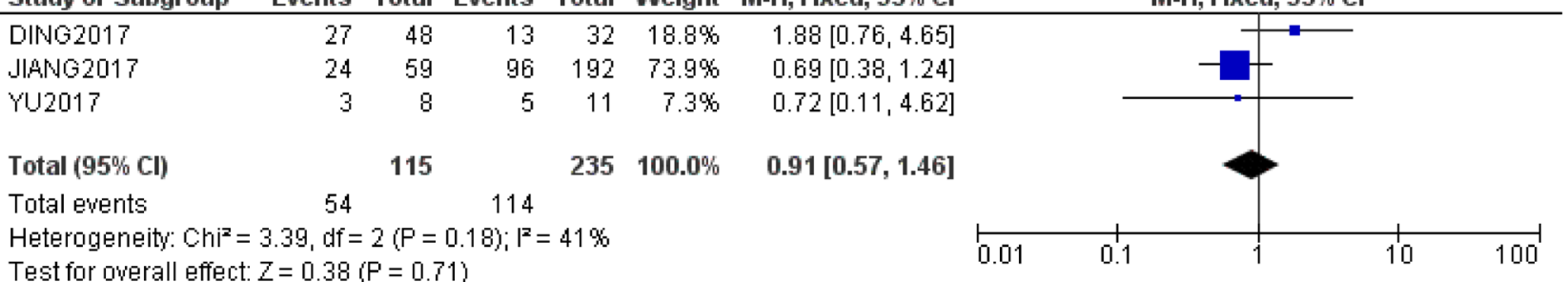

Figure 7. CRNDE and T stage. Forest plot of ORs for the association between CRNDE expression and colorectal cancer T stage.

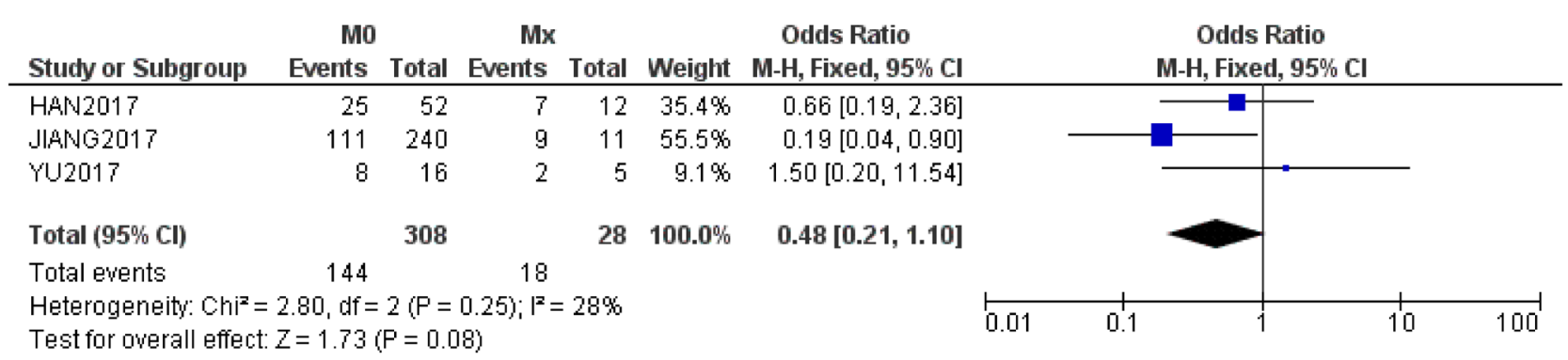

Figure 8. CRNDE and distant metastasis. Forest plot of ORs for the association between CRNDE expression and colorectal cancer distant metastasis.

Publication bias and sensitivity analysis. Figure 9 highlights that funnel plot analysis evaluated publication bias for correlation between CRNDE expression and OS in cancer patients. The results showed that no obvious publication bias was observed in the included meta-analysis studies.

\section{Discussion}

Based on the results of this meta-analysis, CRNDE was confirmed an oncogene. CRNDE up-regulated expression significantly correlated with poor prognosis and advanced 
tumor progression. Moreover, CRNDE has been reported to be involved in the following oncogenic pathways:

(1) Depleted CRNDE in gastric cancer cells results in decreased expression of $\mathrm{p}-\mathrm{PI} 3 \mathrm{~K}$ and $\mathrm{p}$-Akt, thus indicating positive correlation between CRNDE and the PI3K/Akt signaling pathway [8].

(2) The expression of CRNDE in gliomas is believed to be associated with EGFR activation. An EGFR tyrosine kinase inhibitor can block the up-regulation of CRNDE expression, indicating that EGFR signaling may positively regulate CRNDE expression [9]. Depleted CRNDE expression can induce glioma cell growth arrest and cellular apoptosis [9]. A further glioma cell study reported that suppression of CRNDE expression can induce decreased phosphorylation of P70S6K, inhibit expression of c-Myc and cyclin D1 and raise p53 and PTEN expression. This indicates direct correlation between CRNDE and the mTOR signaling pathway [30].

(3) up-regulated CRNDE expression in lung adeno-carcinoma correlates with radio-resistant lung adeno-carcinoma cell lines [11]. CRNDE also interacts with PRC2 and recruits its core component EZH2 to p21 (CDKN1A) promoter regions, repressing p21 transcription. By epigenetically silencing p21, CRNDE contributes to the formation of radio-resistant lung cancer cells [11].
(4) Shao et al. found that CRNDE depletion in renal cancer cells significantly decreased the level of nuclear betacatenin and ectopic CRNDE expression raised it. This shows the positive relation between CRNDE and the Wnt/beta-catenin signaling pathway [31].

Non-coding RNAs are believed to engage in 'cross-talk' with each other, thereby modulating the bioavailability of their respective targets and imposing various levels of post-transcriptional regulation. CRNDE may also recruit microRNAs to participate in cancer development and metastasis. Examples include:

(1) Breast cancer; Huan et al. reported that CRNDE promotes the migration and invasion ability of breast cancer cells by activated $\mathrm{Wnt} / \beta$-catenin signaling through increasing miR-136 expression [7].

(2) Gastric cancer; Hu et al. indicated that CRNDE promotes gastric cancer formation by sponging miR-145 [15].

(3) Colorectal cancer; Han et al. reported that CRNDE promotes the expression of miR-181a-5p in colorectal cancer cells, resulting in inhibited Wnt/beta-catenin signaling [25]. Further, Yu et al. suggested that CRNDE increases proliferation and invasion of colorectal cancer cells via up-regulated expression of transcription factor 7-like 2 (TCF7L2), involving the activation of Wnt/betacatenin signaling through binding miR-217 [23].

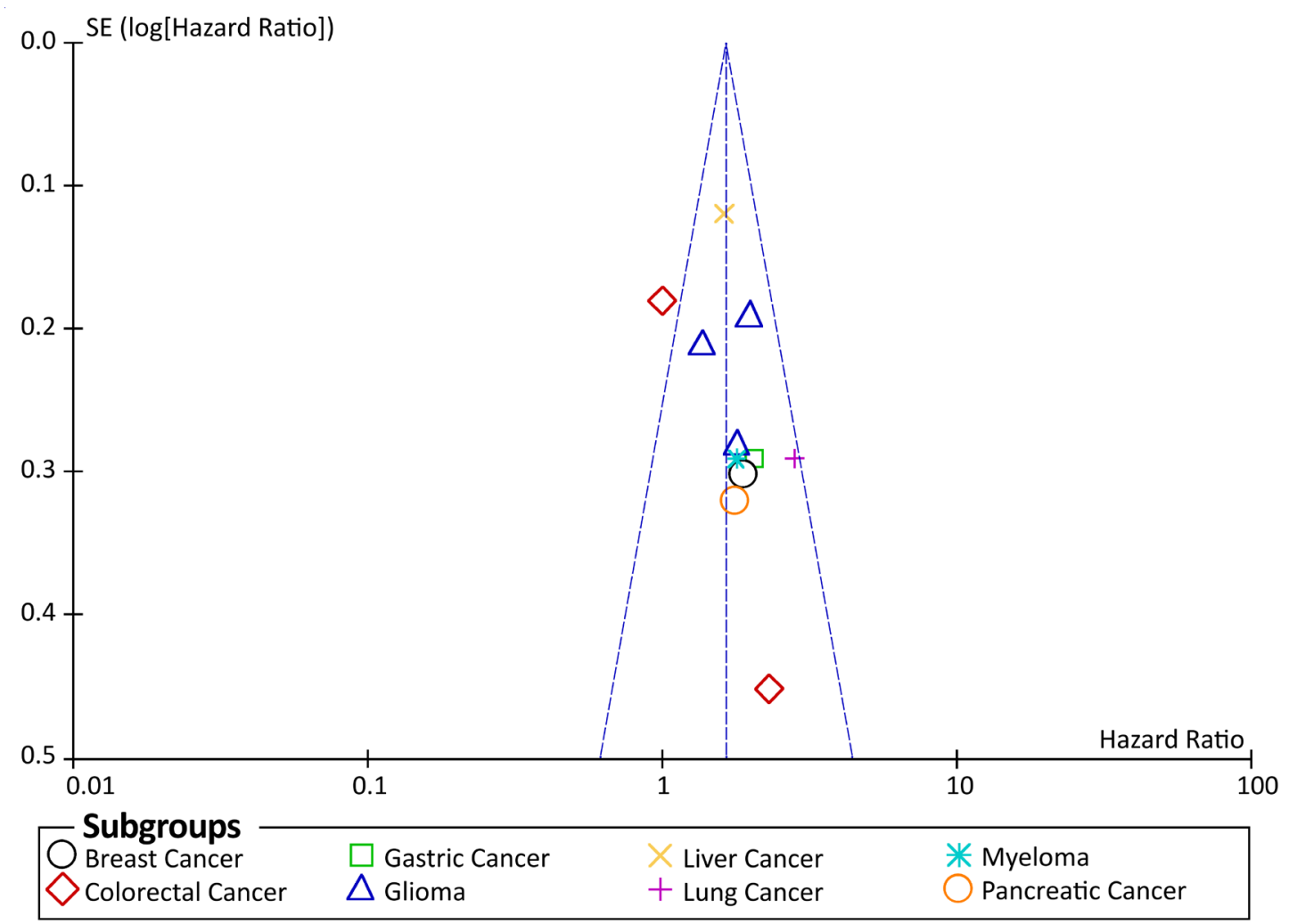

Figure 9. Funnel comparison plot of the association between CRNDE expression and OS. 
(4) Glioma; Zheng et al. showed that CRNDE induces glioma cell proliferation through down-regulated miR-186 expression [32].

(5) Pancreatic cancer; Wang et al. identified that overexpressed CRNDE promotes pancreatic cancer progression through raised IRS1 expression by recruiting miR-384 [13]; and Chen et al. confirmed that CRNDE raises the expression levels of NF-kappa B and p-AKT through depletion of miR-384 [33].

(6) Multiple myeloma; the expression of CRNDE was negatively associated with miR-451 expression; and CRNDE promoted proliferative and anti-apoptotic ability by suppressing miR-451 expression [12].

However, despite successful discovery of CRNDE's oncogenic role in multiple human cancers, there are also some limitations to this systematic review: Most studies were conducted in China and only two in Europe, and the time frames were primarily between 2016 and 2017. The results should therefore be tested over a larger territory and a longer time period. In addition, more parallel studies are required for each kind of human cancer in this review.

Further, some clinical characteristics cannot be normalized due to different study methodology. For example, some studies lacked the cut-off value for CRNDE expression. Moreover, the application of the median value as the cut-off value for high versus low CRNDE expression is not perfect because it does not take the adjacent normal tissues into consideration. Some patients may be placed in the high expression group because of the CRNDE expression levels in tumor tissue, but their adjacent normal tissue may have higher CRNDE expression. These patients should be allocated to the low group instead of the high group. Finally, no prospective study was discovered or included, and this may impair the persuasive capacity of this systematic review to some extent.

In conclusion, this meta-analysis confirms the oncogenic role of CRNDE in several human cancers, and CRNDE will hopefully become a significant prognostic biomarker for cancer patients. Regardless of some limitations, this study is the first systematic review of the prognostic role of CRDDE expression in human cancer, and we look forward to numerous large-scale, multi-center and high-quality prospective studies to fully test our discoveries and support our results.

\section{References}

[1] GONZALEZ CA, AGUDO A. Carcinogenesis, prevention and early detection of gastric cancer: Where we are and where we should go. Int J Cancer 2012; 130: 745-753. https:// doi.org/10.1002/ijc.26430

[2] DJEBALI S, DAVIS CA, MERKEL A, DOBIN A, LASSMANN T et al. Landscape of transcription in human cells. Nature 2012; 489: 101-108. https://doi.org/10.1038/nature 11233
[3] HU X, SOOD AK, DANG CV, ZHANG L. The role of long noncoding RNAs in cancer: the dark matter matters. Curr Opin Genet Dev 2018; 48: 8-15. https://doi.org/10.1016/j. gde.2017.10.004

[4] TAKAHASHI K, YAN I, HAGA H, PATEL T. Long noncoding RNA in liver diseases. Hepatology 2014; 60: 744-753. https://doi.org/10.1002/hep.27043

[5] GRAHAM LD, PEDERSEN SK, BROWN GS, HO T, KASSIR Z et al. Colorectal Neoplasia Differentially Expressed (CRNDE), a Novel Gene with Elevated Expression in Colorectal Adenomas and Adenocarcinomas. Genes Cancer 2011; 2: 829-840. https://doi.org/10.1177/1947601911431081

[6] LIU T, ZHANG X, GAO S, JING F, YANG Y et al. Exosomal long noncoding RNA CRNDE-h as a novel serum-based biomarker for diagnosis and prognosis of colorectal cancer. Oncotarget 2016; 7: 85551-85563. https://doi.org/10.18632/ oncotarget. 13465

[7] HUAN J, XING L, LIN Q, XUI H, QIN X. Long noncoding RNA CRNDE activates Wnt/beta-catenin signaling pathway through acting as a molecular sponge of microRNA-136 in human breast cancer. Am J Transl Res 2017; 9: 1977-1989.

[8] DU DX, LIAN DB, AMIN BH, YAN W. Long non-coding RNA CRNDE is a novel tumor promoter by modulating PI3K/AKT signal pathways in human gastric cancer. Eur Rev Med Pharmacol Sci 2017; 21: 5392-5398. https://doi. org/10.26355/eurrev_201712_13925

[9] KIANG KM, ZHANG XQ, ZHANG GP, LI N, CHENG SY et al. CRNDE Expression Positively Correlates with EGFR Activation and Modulates Glioma Cell Growth. Target Oncol 2017; 12: 353-363. https://doi.org/10.1007/s11523-0170488-3

[10] DAI M, CHEN S, WEI X, ZHU X, LAN F et al. Diagnosis, prognosis and bioinformatics analysis of lncRNAs in hepatocellular carcinoma. Oncotarget 2017; 8: 95799-95809. https://doi.org/10.18632/oncotarget.21329

[11] ZHANG M, GAO C, YANG Y, LI G, DONG J et al. Long Noncoding RNA CRNDE/PRC2 Participated in the Radiotherapy Resistance of Human Lung Adenocarcinoma Through Targeting p21 Expression. Oncol Res 2017. https://doi.org/ 10.3727/096504017X14944585873668[12] MENG YB, HE X, HUANG YF, WU QN, ZHOU YC et al. Long Noncoding RNA CRNDE Promotes Multiple Myeloma Cell Growth by Suppressing miR-451. Oncol Res 2017; 25: 1207-1214. https://doi.org/10.3727/096504017X14886679715637

[13] WANG G, PAN J, ZHANG L, WEI Y, WANG C. Long noncoding RNA CRNDE sponges miR-384 to promote proliferation and metastasis of pancreatic cancer cells through upregulating IRS1 Cell Prolif 2017; 50. https://doi.org/10.1111/ cpr.12389

[14] MENG Y, LI Q, LI L, MA R. The long non-coding RNA CRNDE promotes cervical cancer cell growth and metastasis. Biol Chem 2017; 399: 93-100. https://doi.org/10.1515/ hsz-2017-0199

[15] HU CE, DU PZ, ZHANG HD, HUANG GJ. Long Noncoding RNA CRNDE Promotes Proliferation of Gastric Cancer Cells by Targeting miR-145. Cell Physiol Biochem 2017; 42: 13-21. https://doi.org/10.1159/000477107 
[16] GAO H, SONG X, KANG T, YAN B, FENG L et al. Long noncoding RNA CRNDE functions as a competing endogenous RNA to promote metastasis and oxaliplatin resistance by sponging miR-136 in colorectal cancer. Onco Targets Ther 2017; 10: 205-216. https://doi.org/10.2147/OTT.S116178

[17] ZHENG J, LIU X, WANG P, XUE Y, MA J et al. CRNDE Promotes Malignant Progression of Glioma by Attenuating miR-384/PIWIL4/STAT3 Axis. Molecular therapy 2016; 24: 1199-1215. https://doi.org/10.1038/mt.2016.71

[18] LI Z, TANG Y, XING W, DONG W, WANG Z. LncRNA, CRNDE promotes osteosarcoma cell proliferation, invasion and migration by regulating Notch 1 signaling and epithelialmesenchymal transition. Exp Mol Pathol 2018; 104: 19-25. https://doi.org/10.1016/j.yexmp.2017.12.002

[19] PARMAR MK, TORRI V, STEWART L. Extracting summary statistics to perform meta-analyses of the published literature for survival endpoints. Stat Med 1998; 17: 2815-2834.

[20] DE OLIVEIRA IR, SANTOS-JESUS R, PO AL, POOLSUP N. Extracting numerical data from published reports of pharmacokinetics investigations: method description and validation. Fundam Clin Pharmacol 2003; 17: 471-472.

[21] QI HL, ZHUANG BJ, LI CS, LIU QY. Peri-operative use of sorafenib in liver transplantation: A time-to-event metaanalysis. World J Gastroenterol 2015; 21: 1636-1640. https:// doi.org/10.3748/wjg.v21.i5.1636

[22] TIERNEY JF, STEWART LA, GHERSI D, BURDETT S, SYDES MR. Practical methods for incorporating summary time-to-event data into meta-analysis. Trials 2007; 8: 16. https://doi.org/10.1186/1745-6215-8-16

[23] YU B, YE X, DU Q, ZHU B, ZHAI Q et al. The Long NonCoding RNA CRNDE Promotes Colorectal Carcinoma Progression by Competitively Binding miR-217 with TCF7L2 and Enhancing the Wnt/beta-Catenin Signaling Pathway. Cell Physiol Biochem 2017; 41: 2489-2502. https://doi. org/10.1159/000475941

[24] JIANG H, WANG Y, AI M, WANG H, DUAN Z et al. Long noncoding RNA CRNDE stabilized by hnRNPUL2 accelerates cell proliferation and migration in colorectal carcinoma via activating Ras/MAPK signaling pathways. Cell Death Dis 2017; 8: e2862. https://doi.org/10.1038/cddis.2017.258
[25] HAN P, LI JW, ZHANG BM, LV JC, LI YM et al. The lncRNA CRNDE promotes colorectal cancer cell proliferation and chemoresistance via miR-181a-5p-mediated regulation of Wnt/beta-catenin signaling. Mol Cancer 2017; 16: 9. https://doi.org/10.1186/s12943-017-0583-1

[26] DING J, LI J, WANG H, TIAN Y, XIE $\mathrm{M}$ et al. Long noncoding RNA CRNDE promotes colorectal cancer cell proliferation via epigenetically silencing DUSP5/CDKN1A expression. Cell Death Dis 2017; 8: e2997. https://doi.org/10.1038/ cddis. 2017.328

[27] Jing SY, Lu YY, Yang JK, Deng WY, Zhou Q, Jiao BH. Expression of long non-coding RNA CRNDE in glioma and its correlation with tumor progression and patient survival. Eur Rev Med Pharmacol Sci 2016; 20: 3992-3996.

[28] MURAT A, MIGLIAVACCA E, GORLIA T, LAMBIV WL, SHAY T et al. Stem cell-related "self-renewal" signature and high epidermal growth factor receptor expression associated with resistance to concomitant chemoradiotherapy in glioblastoma. J Clin Oncol 2008; 26: 3015-3024. https://doi. org/10.1200/JCO.2007.15.7164

[29] GRAVENDEEL L, KOUWENHOVEN M, GEVAERT $\mathrm{O}$, DE ROOI J, STUBBS A et al. Intrinsic gene expression profiles of gliomas are a better predictor of survival than histology. Cancer Res 2009; 69: 9065-9072. https://doi. org/10.1158/0008-5472.CAN-09-2307

[30] WANG Y, WANG Y, LI J, ZHANG Y, YIN H et al. CRNDE, a long-noncoding RNA, promotes glioma cell growth and invasion through mTOR signaling. Cancer Lett 2015; 367: 122-128. https://doi.org/10.1016/j.canlet.2015.03.027

[31] SHAO K, SHI T, YANG Y, WANG X, XU D et al. Highly expressed lncRNA CRNDE promotes cell proliferation through Wnt/beta-catenin signaling in renal cell carcinoma. Tumour Biol 2016. https://doi.org/10.1007/s13277-016-5440-0

[32] ZHENG J, LI XD, WANG P, LIU XB, XUE YX et al. CRNDE affects the malignant biological characteristics of human glioma stem cells by negatively regulating miR-186. Oncotarget 2015; 6: 25339-25355. https://doi.org/10.18632/oncotarget. 4509

[33] CHEN Z, YU C, ZHAN L, PAN Y, CHEN L et al. LncRNA CRNDE promotes hepatic carcinoma cell proliferation, migration and invasion by suppressing miR-384. Am J Cancer Res 2016; 6: 2299-2309. 